\title{
Análise Comparativa de Versões YOLO na Detecção e Identificação de Parasitas da Malária
}

\author{
Maura G. R. Rocha ${ }^{1}$, Rodrigo M. S. Veras ${ }^{1}$, Maíla L. Claro ${ }^{1}$, Laurindo S. Britto Neto ${ }^{1}$, \\ Kelson R. T. Aires ${ }^{1}$ \\ ${ }^{1}$ Departamento de Computação - Universidade Federal do Piauí (UFPI) \\ Teresina, Brasil \\ \{mrocha, rveras, claromaila, laurindoneto, kelson\}@ufpi.edu.br
}

\begin{abstract}
Malaria is an endemic disease caused by the parasite Plasmodium that can be fatal in many regions of the world. Some researchers use machine learning concepts to detect and classify cells infected by the Plasmodium parasite. In this work, we compared three recent versions of the convolutional neural network You Only Look Once (YOLO): YOLOv4, Scaled-YOLOv4, and YOLOv5. We used the MP-IDB dataset, which has 210 images with the parasite Plasmodium. The models achieved excellent results, having the best result with $m A P$ and accuracy of $94.8 \%$ and $93.3 \%$, respectively, for the classification in two types of species of Plasmodium falciparum and vivax.
\end{abstract}

Resumo. A malária é uma doença endêmica causada pelo parasita Plasmodium que pode ser fatal em muitas regiões do mundo. Alguns pesquisadores estão utilizando conceitos de aprendizagem de máquina para detectar e classificar células infectadas pelo parasita Plasmodium. Este trabalho apresenta um estudo comparativo de três versões recentes da rede neural convolucional You Only Look Once (YOLO), são elas a: YOLOv4, Scaled-YOLOv4 e YOLOv5. Foi utilizado a base de dados MP-IDB que possui 210 imagens com o parasita Plasmodium. Os modelos alcançaram excelentes resultados, tendo o meIhor resultado com mAP e precisão de 94,8\% e 93,3\%, respectivamente, para a classificação em dois tipos de espécies do Plasmodium falciparum e vivax.

\section{Introdução}

A malária é uma doença infecciosa causada pela picada da fêmea do mosquito Anopheles, infectado pelo parasita do gênero Plasmodium. Essa doença é considerada um grave problema de saúde pública no mundo. De acordo com o Relatório Mundial da Malária 2020 da Organização Mundial da Saúde (OMS), quase a metade da população mundial vive em áreas de risco de transmissão. Em 2019, a malária causou cerca de 229 milhões de casos e 409 mil mortes. Estima-se que $94 \%$ das mortes em 2019 ocorreram no continente africano [Organization et al. 2020].

As principais espécies de Plasmodium que infectam os seres humanos são: malariae (P. malariae), ovale ( $P$. ovale), falciparum ( $P$. falciparum) e vivax ( $P$. vivax), sendo essas duas últimas espécies as mais comuns [Organization et al. 2020]. A Figura 1 apresenta exemplos de imagens dessas espécies, que diferem nas mudanças das células infectadas, forma, presença de pontos característicos e morfologia do parasita, em alguns dos 


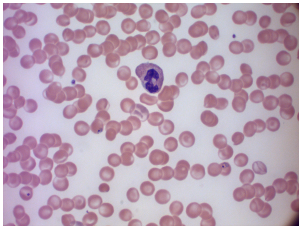

(a) P. falciparum.

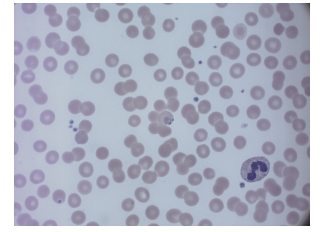

(b) P. malariae.

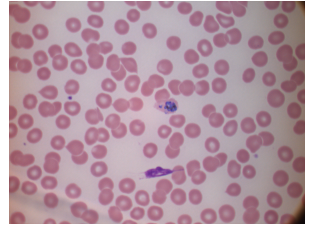

(c) P. ovale.

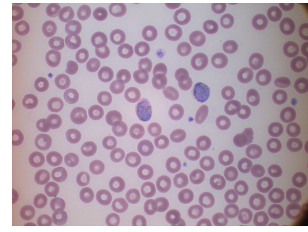

(d) P. vivax.

Figura 1. Exemplos de imagens das principais espécies de Plasmodium.

estágios do ciclo de vida [Somasekar et al. 2011]. Os principais estágios de desenvolvimento do parasita da malária são: ring, trophozoite, schizont e gametocytes.

Existem várias técnicas para diagnosticar a malária. Contudo, a microscopia manual continua sendo a principal técnica em países subdesenvolvidos, devido ao seu baixo custo e capacidade de detectar as espécies. Embora esse processo seja considerado o padrão ouro para o diagnóstico, essa técnica requer pessoas treinadas e experientes no exame de distensões sanguíneas. Além disso, esse tipo de inspeção visual é demorado e está sujeito a erro humano, causados por fadiga ou trabalho repetitivo [Ramarolahy et al. 2020]. A fim de superar essas dificuldades, diversos sistemas de Diagnóstico Auxiliado por Computador (Computer Aided Diagnosis - CAD) têm sido propostos para a detecção e classificação automática do parasita da malária em imagens de esfregaços de sangue.

Este estudo apresenta técnicas automatizadas para a detecção do parasita Plasmodium da malária e classificação de espécies a partir de imagens de esfregaço de sangue fino, proporcionando um diagnóstico mais preciso, ágil e com custos reduzidos. Para isso, o estudo propõe análise comparativa das versões do modelo You Only Look Once (YOLO) [Redmon et al. 2016] - YOLOv4, Scaled-YOLOv4 e YOLOv5, aplicadas a 210 imagens que possuem as quatro espécies de parasitas.

O presente artigo está organizado da seguinte forma: a Seção 2 apresenta os trabalhos relacionados referentes à detecção da malária; a Seção 3 fornece informações detalhadas sobre os materiais e métodos utilizados neste estudo, como base de imagens, o modelo YOLO e as métricas de avaliação; a Seção 4 apresenta os resultados obtidos e suas discussões; e a Seção 5 apresenta a conclusão e os trabalhos futuros.

\section{Estado da Arte}

Essa seção apresenta um levantamento sistemático do estado da arte relacionado ao diagnóstico auxiliado por computador para detecção e classificação de células infectadas pelo parasita da malária. A pesquisa teve como objetivo identificar e classificar os trabalhos disponíveis na literatura com base nas técnicas utilizadas, as bases de imagens aplicadas e a relevância.

A pesquisa foi realizada usando três conjuntos de dados públicos: Scopus, SpringerLink e IEEE Xplore. As seguintes strings de busca foram aplicadas: detection of malaria, detection and classification of malaria e blood smear malaria detection. Em seguida, foi selecionando os trabalhos publicados após 2015 na área da computação. No banco de dados Scopus a consulta retornou 37 trabalhos, o banco de dados IEEE Xplorer retornou 24 e o SpringerLink retornou 42 trabalhos. Após a leitura do resumo e do texto completo dos artigos, aplicou-se critérios de exclusão para seleção de artigos que são per- 
tinentes ao tema do presente estudo. Assim, obteve-se 9 artigos, dividindo eles em artigos que realizaram a classificação do parasita com métodos diferentes do YOLO e métodos que aplicam a rede YOLO.

Peñas et al. 2017 implementaram um sistema para detectar a presença de parasitas da malária e identificar suas espécies em imagens de amostras de sangue. Os testes foram realizados com base de dados privada que possui 363 imagens. Para realizar a segmentação do parasita, utilizou-se a análise de componentes conectados. Para classificar os parasitas, utilizou-se a Rede Neural Convolucional (Convolutional Neural Network - CNN) InceptionV3. Na detecção do parasita alcançaram uma acurácia de 92,40\% e para classificação das espécies em $P$. vivax e $P$. falciparum uma acurácia de 87,90\%.

Em Maity et al. 2020, os autores propuseram uma estrutura de aprendizado de máquina para a segmentação de células sanguíneas infectadas com parasitas da malária, além da classificação das espécies e estágios desses parasitas. Nesse estudo foram utilizadas duas espécies específicas: $P$. vivax e $P$. falciparum, com os seus estágios de vida sendo considerados para classificação. A segmentação foi baseada em pixels regulada por classificador supervisionado. O processo de classificação foi realizado utilizando a rede de Capsule Network (CapsNet). A segmentação alcançou 99,1\% de acurácia e a classificação alcançou 98,7\% de acurácia.

Os trabalhos de Cruz et al. 2020, Alqudah et al. 2020 e Maqsood et al. 2021 propuseram novas arquiteturas de CNN para classificação de células sanguíneas infectadas pelo P.falciparum. A base de dados utilizada por esses trabalhos foi o NIH Malaria fornecido pela National Library of Medicine ${ }^{1}$ [Rajaraman et al. 2018]. Tal conjunto de dados contém 27.558 imagens de células com instâncias iguais de células parasitadas e não infectadas. Os resultados dos testes mostraram que Cruz et al. 2020, Alqudah et al. 2020 e Maqsood et al. 2021 alcançaram acurácia, respectivamente, de 98,88\%, 98,85\% e $96,82 \%$ na classificação. Existem outros estudos que utilizam a mesma base de dados para os seus métodos com aplicação de CNNs. Contudo, foi selecionado apenas os três estudos mais recentes, que apresentaram o melhor desempenho.

Em Rahman et al. 2021, os autores avaliaram o desempenho de várias arquiteturas de CNNs pré-treinadas para classificação de células sanguíneas infectadas pelo Plasmodium. Esse trabalho utilizou duas bases de dados. O primeiro conjunto foi o BBBC041v1 [Ljosa et al. 2012], que consiste em 1.364 imagens de P.Falciparum e P.vivax. Para o experimento, foi realizado a binarização de todo o conjunto de dados e o dividiram em duas classes: 56.149 células não infectadas e 2.099 células infectadas. O segundo conjunto é o MP-IDB que foi usado para realizar testes independentes em todos os classificadores. O mesmo procedimento foi realizado nesse segundo conjunto de dados. Os resultados dos testes mostraram que classificador com o melhor desempenho em termos de acurácia e F1-score na base de dados BBBC041v1 foi TL-NIH com 99,35\% e $96,82 \%$, respetivamente, e no conjunto MP-IDB o classificador VGG-19 com 85,18\% de acurácia e $84,82 \%$ de F1-score.

Nosso estudo investiga a aplicação da rede YOLO, que é um sistema para detecção de classes de objetos em tempo real. Por isso, foi realizado uma busca por trabalhos que utilizam essa rede. Foram encontrados três trabalhos que serão descritos a seguir.

\footnotetext{
${ }^{1}$ https://lhncbc.nlm.nih.gov
} 
No trabalho de Yang et al. 2020a, os autores desenvolveram um sistema de diagnóstico para a detecção automatizada de parasitas $P$. vivax usando um modelo Cascaded YOLO. Esse modelo consiste no modelo YOLOv2 e um classificador hard-negative mining. Foram feitos testes em 2.567 imagens de esfregaço de sangue fino de 171 pacientes e os resultados demonstraram uma eficácia desse modelo desenvolvido em relação ao modelo YOLOv2, tendo o mean Average Precision (mAP) de 79,22\%.

Chibuta and Acar 2020 desenvolveram um pipeline de processamento de imagem para detectar o P. falciparum usando um algoritmo de detecção YOLOv3 modificado. Os testes foram realizados em dois conjuntos de dados. O primeiro conjunto (Dataset A) ${ }^{2}$ tem 2.703 imagens e foi coletado usando uma câmera de microscópio, em que os autores obtiveram uma precisão e acurácia de 99,17\% e 99,07\%, respectivamente. Já segundo conjunto (Dataset B) ${ }^{3}$ tem 1.182 imagens e foi coletado usando uma câmera de telefone móvel, em que os autores encontraram uma precisão e acurácia de 96,65\% e 97,46\%, respectivamente.

Abdurahman et al. 2021 investigaram os modelos YOLOv3 e YOLOv4 para detectar e classificar automaticamente parasitas da malária a partir de imagens microscópicas de lâminas de sangue. Os autores realizaram algumas modificações nesses dois modelos e alcançaram um melhor desempenho de detecção em comparação com suas versões originais. O melhor resultado encontrado foi com YOLOV4-MOD @608 × 608, com mAP de 96,32\% no Dataset A. A Tabela 1 apresenta um resumo dos trabalhos do estado da arte.

Tabela 1. Resumo dos trabalhos encontrados na literatura.

\begin{tabular}{|c|c|c|c|c|c|}
\hline \multicolumn{6}{|c|}{ Detecção e Classificação com CNNs } \\
\hline Autores & Métodos & Classificação & Base de Dados & $\mathrm{N}^{\mathbf{0}}$ de Imagens & Acurácia(\%) \\
\hline Peñas et al. 2017 & CNN InceptionV3 & $\begin{array}{c}P . \text { vivax } \times \\
\text { P. falciparum }\end{array}$ & Privada & 363 & 87,90 \\
\hline Maity et al. 2020 & CNN Capsnet & $\begin{array}{c}\text { P. vivax Ring } \times \\
\text { P. vivax Gametocyte } \times \\
\text { P. vivax Schzonat } \times \\
\text { P. falciparum Ring }\end{array}$ & $\begin{array}{c}\text { MP-IDB } \\
\text { Privada }\end{array}$ & 248 & $\begin{array}{c}\text { Segm: } 99,10 \\
\text { Clas: } 98,70\end{array}$ \\
\hline Cruz et al. 2020 & CNN proposta & $\begin{array}{l}P \text {. falciparum } \times \\
\text { Não parasitada }\end{array}$ & NIH Malaria & 27.558 & 98,88 \\
\hline Alqudah et al. 2020 & CNN proposta & $\begin{array}{l}P \text { falciparum } \times \\
\text { Não parasitada }\end{array}$ & NIH Malaria & 27.558 & 98,85 \\
\hline Maqsood et al. 2021 & CNN proposta & $\begin{array}{l}P \text { falciparum } \times \\
\text { Não parasitada }\end{array}$ & NIH Malaria & 27.558 & 96,82 \\
\hline \multicolumn{6}{|c|}{ Detecção e Classificação com YOLO } \\
\hline Autores & Métodos & Classificação & Base de Dados & $\mathrm{N}^{\mathbf{0}}$ de Imagens & Resultado(\%) \\
\hline Yang et al. 2020a & Cascaded YOLO & P. vivax & Privada & 2.567 & mAP: 79,22 \\
\hline Chibuta and Acar 2020 & YOLOv3 Modificada & P. falciparum & $\begin{array}{l}\text { Dataset } A \\
\text { Dataset B }\end{array}$ & $\begin{array}{l}2.703 \\
1.182\end{array}$ & $\begin{array}{l}\text { A: } 99,07 \\
\text { A: } 97,46\end{array}$ \\
\hline Abdurahman et al. 2021 & YOLOv4-MOD & P. falciparum & Dataset A & 1.182 & mAP: 96,32 \\
\hline
\end{tabular}

\footnotetext{
${ }^{2}$ http://air.ug/downloads/plasmodium-images.zip

${ }^{3}$ http://air.ug/downloads/plasmodium-phonecamera.zip
} 


\section{Materiais e Métodos}

O objetivo deste trabalho consiste em detectar o parasita Plasmodium da malária nas imagens de esfregaço de sangue fino. Em seguida, será feita a classificação das espécies em facilparum, vixax, malariae e ovale. Para isso, foi utilizado algumas versões do modelo You Only Look Once (YOLO) [Redmon et al. 2016], que é uma CNN baseada em Region of Interest (ROI). De acordo com estudos da literatura [Al-Masni et al. 2018, Nie et al. 2019, Yang et al. 2020a, Chibuta and Acar 2020, Abdurahman et al. 2021], tal modelo apresentou excelentes resultados realizando a detecção e classificação simultaneamente.

\subsection{Base de Imagens}

Neste estudo foi utilizado o conjunto de dados Malaria Parasite Image Database (MPIDB) [Loddo et al. 2018] para detecção e classificação do parasita da malária. O MP-IDB é um conjunto de imagens públicas de amostras de sangue periférico composto por 210 imagens com resolução de $2592 \times 1944$ pixels, classificadas em quatro tipos diferentes de Plasmodium: facilparum, vixax, malariae e ovale. A Tabela 2 descreve a quantidade de imagens e de parasitas por espécies.

Tabela 2. Composição da base de imagens MP-IDB.

\begin{tabular}{ccc}
\hline Espécies & Quantidade de imagens & Quantidade de parasitas \\
\hline Plasmodium facilparum & 104 & 1198 \\
\hline Plasmodium vivax & 40 & 62 \\
\hline Plasmodium malariae & 37 & 43 \\
\hline Plasmodium ovale & 29 & 33 \\
\hline
\end{tabular}

Cada imagem contém pelo menos um parasita em diferentes fase da vida: anel, trofozoíta, esquizontes e gametócitos. A Figura 2 apresenta exemplos de imagens com os tipos de parasita do Plasmodium e em destaque a espécie do parasita.

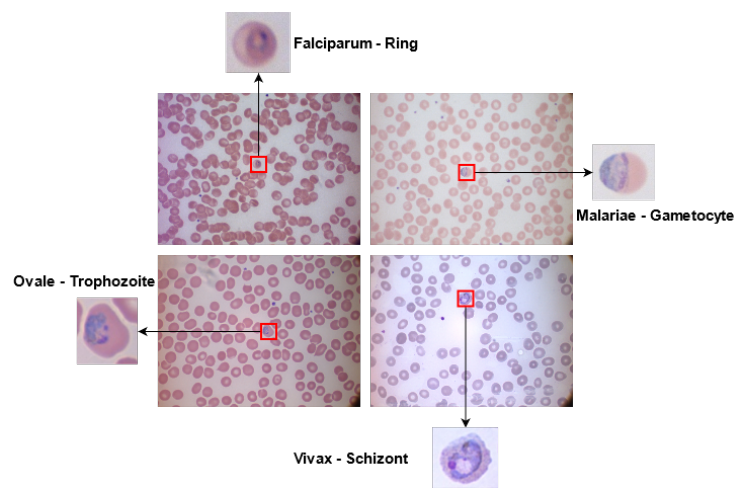

Figura 2. Tipos de Plasmodium: sentido horário a partir do canto superior esquerdo, $P$. falciparum em ring, $P$. malariae em gametocyte, $P$. vivax em schizont e $P$. ovale em trophozoite.

\subsection{Versões da YOLO Avaliadas}

A rede YOLO é um dos métodos de aprendizagem profunda, que pode realizar a detecção de objetos em tempo real com uma boa precisão [Redmon et al. 2016]. Essa rede é capaz de realizar a detecção e classificação de vários objetos em uma imagem em tempo real usando uma única $\mathrm{CNN}$ como extrator de características. O funcionamento dessa 
rede consiste em dividir a imagem de entrada em sub-regiões e prevê várias caixas delimitadoras e as pontuações de confiança para cada região [Redmon et al. 2016]. Essas pontuações de confiança são a expressão da existência ou ausência de qualquer objeto dentro da caixa [Ünver and Ayan 2019].

Essa rede tem várias versões com diferentes melhorias como tempo de execução, tamanho e número de objetos que podem ser detectados com uma boa precisão. Esse estudo realiza um comparativo de três versões: YOLOv4, Scaled-YOLOv4 e YOLOv5.

No modelo YOLOv4 foi introduzido o aprimoramento de dados em mosaico no processamento. Além disso, a função de ativação e a função de perda foram otimizados, o que tornou a rede YOLOv4 mais rápida e alcançou um melhor equilíbrio entre a precisão e tempo de execução do que as versões anteriores. A arquitetura desse modelo usa: CSPDarknet53 baseando em CSPNet, que é uma estrutura de rede neural de código aberto, como a principal rede de backbone para treinar e extrair características de imagem; neck usa Spatial Pyramid Pooling (SPP) e Path Aggregation Network (PAN) para obter uma melhor fusão das características extraídas. Além disso, usa o heard que explora o mesmo que a rede YOLOv3 para realizar a detecção de objetos [Bochkovskiy et al. 2020].

YOLOv5 é a primeira implementação nativa do PyTorch e enfatiza a facilidade de uso, a rapidez de treinamento a inferência. Sua arquitetura consiste em três partes: backbone, neck e heard [Zhou et al. 2021]. YOLOv5 combina as funções de YOLOv4, ou seja, adicionando SPP-NET, ao mesmo tempo, propõe novos métodos de aprimoramento de dados, com treinamento mosaic, treinamento auto-antagônico (SAT) e PANet [Liu et al. 2018] em vez de Feature Pyramid Network (FPN) [Yang et al. 2020b].

O Scaled-YOLOv4 é um detector de um estágio de última geração que é aplicável a redes pequenas e grandes, mantendo a velocidade e a precisão na detecção de objetos. Ele é implementado no framework YOLOv5. Esse modelo usa em backbone o CrossStage Partial Networks (CSP) em sua rede. CSP é uma nova maneira de arquitetar CNNs que economiza computação para várias outras redes [Wang et al. 2020].

\subsection{Configurações de Treinamento}

Para o refinamento dos modelos ser mais rápido, todas as imagens do conjunto de dados foram redimensionadas para a resolução de $416 \times 416$. Em seguida, cada imagem foi rotulada de acordo com as necessidades da rede YOLO. Essa rede necessita de algumas informações, como a coordenada do ponto médio $(\mathrm{x}, \mathrm{y})$ do parasita na imagem, valores de largura e altura da caixa delimitadora e sua definição da classe do objeto a ser detectado. Para isso, uma caixa delimitadora foi desenhada ao redor do parasita usando ferramenta labellmg ${ }^{4}$. O tamanho da caixa delimitadora foi definida com base na verdade fundamental fornecida na base de imagens. Assim, todas as anotações das localizações dos parasitas foram formatadas em arquivos $\mathrm{xml} \mathrm{com}$ o formato de dados YOLO.

As imagens do conjunto de dados MP-IDB foram divididas em $70 \%$ para treinamento, $20 \%$ para validação e $10 \%$ para teste. As imagens foram divididas aleatoriamente, mantendo o equilíbrio entre a quantidade de imagens por espécies. O conjunto de dados de treinamento é um grupo de dados usado para treinar o modelo; o conjunto de dados de validação é utilizado durante o treinamento para ter uma ideia de como o modelo estava se

\footnotetext{
${ }^{4}$ https://github.com/tzutalin/labelImg
} 
saindo nas imagens que não estavam sendo usadas no treinamento; e o conjunto de dados de teste foi uma amostra de dados usada para fornecer uma avaliação do desempenho do modelo final.

No refinamento desses modelos utilizou-se os pesos pré-treinados do conjunto de dados MS COCO [Lin et al. 2014] de 80 classes. Os outros hiperparâmetros foram definidos de acordo com as características da imagem do conjunto de dados malária e o desempenho da Graphics Processing Unit (GPU) com um melhor efeito de treinamento: a taxa de aprendizado inicial de 0,001; batch size foi de 64 para o modelo YOLOv4 e 16 para os modelos Scaled-YOLOv4 e YOLOv5; o momentum foi igual a 0,949 e decay igual a 0,0005. A Tabela 3 demonstra o número de épocas dos modelos YOLOv4, Scaled-YOLOv4 e YOLOv5 para o treinamento das classes em quatro e duas espécies de parasitas. A quantidade de épocas foi definida de acordo com os critérios de perda do treinamento e as métricas de desempenho no conjunto de validação. Foi usado a métrica mAP para obter a indicação de boa combinação de hiperparâmetros. Essa métrica é usada para avaliar o desempenho do treinamento, quanto maior for o valor de mAP melhor é o resultado do treinamento.

Tabela 3. Número de épocas de treinamento de acordo com a quantidade (Qt) de classes.

\begin{tabular}{ccc}
\hline & Qt de Classes: 4 & Qt de Classes: 2 \\
\hline Modelo & Número de épocas & Número de épocas \\
\hline YOLOv4 & 8000 & 4000 \\
\hline Scaled-YOLOv4 & 500 & 1000 \\
\hline YOLOv5 & 4000 & 2000 \\
\hline
\end{tabular}

\subsection{Métricas de Avaliação}

A avaliação do desempenho das redes YOLO foi calculada com base nos valores obtidos pela matriz de confusão. Com base na matriz, obtemos quatro valores, denominados Verdadeiro Positivo (VP), Falso Positivo (FP), Falso Negativo (FN) e Verdadeiro Negativo $(\mathrm{VN})$. Com esses valores são calculados as seguintes métricas: Precisão $(P), \operatorname{Recall}(R)$ e Fl-score (Fl-score) segundo as equações 1, 2 e 3, respectivamente.

$$
\begin{gathered}
P=\frac{V P}{V P+F P} \\
R=\frac{V P}{V P+F N} \\
\text { F1-score }=2 * \frac{P * R}{P+R}
\end{gathered}
$$

Outra métrica utilizada foi a mean Average Precision (mAP), que corresponde ao valor médio da precisão. Sua fórmula é apresentada na Equação 4, em que $C$ é o número de classes de parasitas e Average Precison (AP) é a área da curva de precisão versus recall, também chamada de curva AP.

$$
\mathrm{mAP}=\frac{\sum_{i=1}^{C} A P i}{C}
$$


Assim, o mAP é o valor médio do AP quando um parasita de malária é detectado; e quanto maior esse valor, melhor será o resultado da detecção de um parasita da malária. Essa métrica compara cada classe, para cada caixa delimitadora no resultado da detecção, classificada por confiança, e calcula a Intersecção sobre a União (IoU) com um objeto que tem o mesmo rótulo. Neste estudo, consideramos os parasita detectados corretamente se a IoU for igual ou superior a 50\%, assim como em trabalhos encontrados na literatura [Nie et al. 2019, Al-Masni et al. 2018, Wu et al. 2020].

\section{Resultados e Discussões}

Neste trabalho, foram realizados dois testes com a base de dados MP-IDB. O primeiro teste com as quatro espécies, $P$. falciparum, $P$. vivax, $P$. malariae e $P$. ovale, consiste em 210 imagens com 1.336 parasitas. O segundo teste foi só com duas espécies: P. falciparum e $P$ vivax porque são mais predominantes e que causam inúmeras mortes no mundo, para esse teste foi utilizado 144 imagens com 1.260 parasitas.

As Tabelas 4 e 5 apresentam os resultados de desempenho dos testes com quatro classes (P. falciparum - PF, $P$. vivax - PV, P. malarie - PM e P. ovale - PO) e duas classes (PF e PV), respectivamente. Os resultados descritos nessas tabelas demostraram que o modelo YOLOv5 obteve um melhor desempenho de detecção e classificação dos parasitas do que os modelos YOLOv4 e Scaled-YOLOv4, tendo uma precisão de 87,9\% e 93,3\%, e mAP de $93,7 \%$ e $94,8 \%$ para a classificação em quatro e duas espécies, respectivamente. A Figura 3 mostra a curva de precisão $\times$ recall obtida para os testes com quatro e duas classes com o modelo YOLOv5.

Tabela 4. Resultados dos modelos avaliados para detecção e classificação do $P$. falciparum, P. vivax, P. malariae e $P$. ovale.

\begin{tabular}{ccccc}
\hline Modelo & P (\%) & R (\%) & F1-score (\%) & mAP (\%) \\
\hline YOLOv4 & 85 & 88 & 86 & 84,51 \\
\hline Scaled-YOLOv4 & 42,3 & 96,7 & 58,85 & 91 \\
\hline YOLOv5 & $\mathbf{8 7 , 9}$ & $\mathbf{9 0}$ & $\mathbf{8 8 , 9}$ & $\mathbf{9 3 , 7}$ \\
\hline
\end{tabular}

Tabela 5. Resultados dos modelos avaliados para detecção e classificação do $P$. falciparum e $P$. vivax.

\begin{tabular}{ccccc}
\hline Modelo & P (\%) & R (\%) & F1-score (\%) & mAP (\%) \\
\hline YOLOv4 & 87 & 93 & 90 & 95,27 \\
\hline Scaled-YOLOv4 & 64,4 & 94,2 & 76,5 & 88,2 \\
\hline YOLOv5 & $\mathbf{9 3 , 3}$ & $\mathbf{9 4 , 2}$ & $\mathbf{9 3 , 7}$ & $\mathbf{9 4 , 8}$ \\
\hline
\end{tabular}

Outro ponto a ser destacado é que a Tabela 5 apresenta melhores resultados em todos os modelos quando comparados com a Tabela 4 . O experimento com quatro classe é mais complexo computacionalmente que com duas classes. Na Tabela 5, pode-se perceber que os modelos YOLOv4 e YOLOv5 alcançaram alta taxa de detecção com mAP de 95,27\% e 94,8\%, respectivamente, e YOLOv5 apresentou o melhor desempenho com precisão $(93,3 \%)$, recall $(94,2 \%)$ e $F 1$-score $(93,7 \%)$.

Os resultados mostraram que o modelo YOLOv5 obteve melhores resultados tanto no teste de quatro classes quanto no teste de duas classes. Esse modelo apresentou um menor tempo de inferência de detecção com valor de 9 milissegundos (ms) por imagem, 


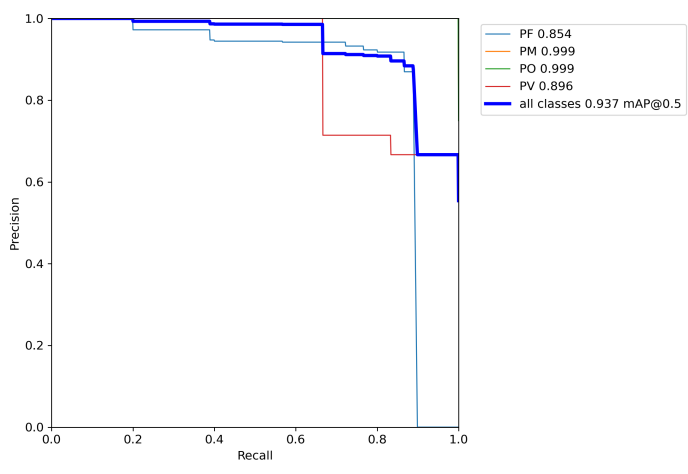

(a)

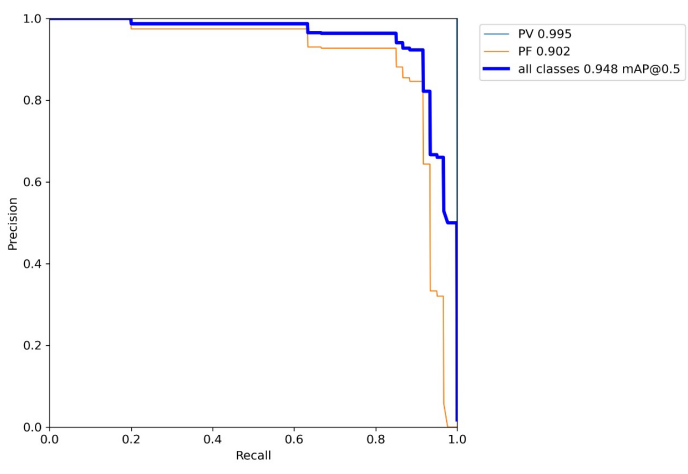

(b)

Figura 3. Curva de precisão $\times$ recall do modelo YOLOv5: (a) quatro classes, (b) duas classes.

em comparação aos modelos YOLOv4 e Scaled-YOLOv4, que levaram $40 \mathrm{~ms}$ e $21 \mathrm{~ms}$, respectivamente. YOLOv5 é escrito na estrutura Ultralytics PyTorch ${ }^{5}$, que faz de inferências, ou seja, a detecção do parasita, mais rápido do que os concorrentes por ser uma estrutura de tamanho menor. Além disso, esse modelo usa aumento de mosaico em seu treinamento, que combina quatro imagens em quatro blocos de proporção aleatória. $\mathrm{O}$ aumento do mosaico é especialmente útil para detecção de objetos, ajudando o modelo a aprender a lidar com objetos pequenos.

A Figura 4 demonstra exemplos da detecção e classificação de parasitas em imagem de esfregaço de sangue. As caixas coloridas nas imagens mostram os parasitas detectados e valor de confiança do parasita ser de determinada classe. Na Figura 4, todos os parasitas foram detectados corretamente para as quatro espécies pelo modelo YOLOv5.

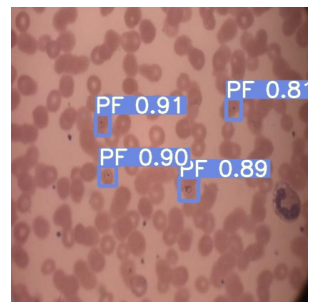

(a) P. falciparum

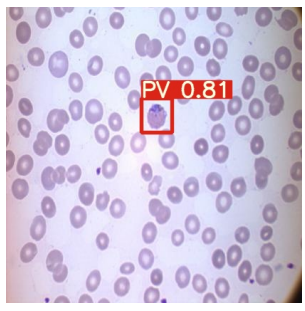

(b) P. vivax

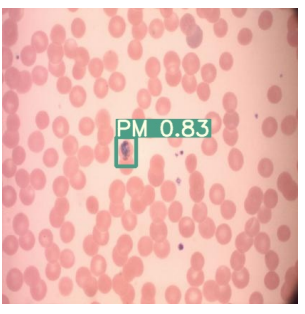

(c) P. malariae

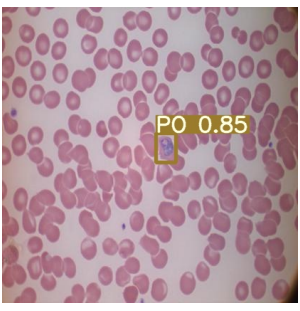

(d) P. ovale

Figura 4. Resultados da detecção e classificação dos parasitas pelo YOLOV5.

Apesar dos bons resultados, ainda ocorrem erros. A Figura 5 apresenta exemplos específicos em que os parasitas não foram detectados e classificados corretamente. Os casos de erros estão marcados com uma seta vermelha.

Esses erros, de detecção e classificação dos parasitas, que são vistos na Figura 5, foram ocasionados principalmente pela quantidade de imagens por classe ser pequena, o que causa uma diminuição na qualidade do treinamento. Além disso as imagens de esfregaço de sangue do banco de dados MP-IDB possuem diferentes condições de iluminação e coloração, dificultando ainda mais a detecção do parasita.

\footnotetext{
${ }^{5}$ https://github.com/ultralytics
} 


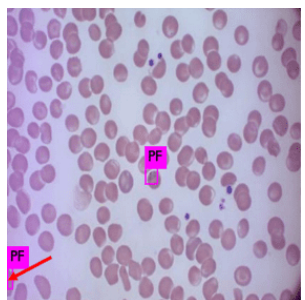

(a)

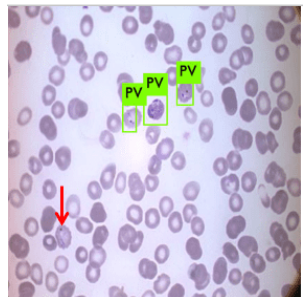

(b)

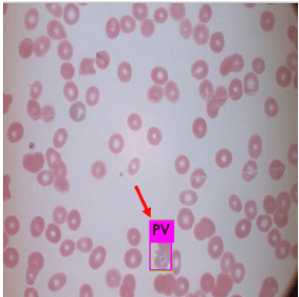

(c)

Figura 5. Exemplos de detecção e classificação errôneas em diferentes espécies de parasitas: (a) representa falso positivo, (b) representa falso negativo e (c) exemplo em que o modelo classificou o mesmo parasita para duas classes (PV e PO), porém o correto é PV.

A Tabela 6 apresenta uma comparação do desempenho da rede YOLOv5 com os trabalhos do estado da arte, seguindo o critério de que os trabalhos utilizaram o mesmo conjunto de dados de imagens (MP-IDB).

Tabela 6. Comparação dos resultados obtidos com os trabalhos relacionados.

\begin{tabular}{|c|c|c|c|c|}
\hline Autores & Métodos & Classificação & Qt de Imagens & Acurácia(\%) \\
\hline Maity et al. 2020 & CNN Capsnet & $\begin{array}{c}\text { P. vivax Ring } \times \\
\text { P. vivax Gametocyte } \times \\
\text { P. vivax Schzonat } \times \\
\text { P. falciparum Ring }\end{array}$ & 100 & $\begin{array}{l}\text { Segm: } 97,1 \\
\text { Clas: } 96,6\end{array}$ \\
\hline Rahman et al. 2021 & Arquiteturas $\mathrm{CNN}$ & $\begin{array}{l}\text { Plasmodium } \times \\
\text { Não parasitada }\end{array}$ & 210 & 85,18 \\
\hline Modelo avaliado & YOLOv5 & $\begin{array}{c}\text { P. vivax } \times \\
\text { P. falciparum }\end{array}$ & 144 & 91,10 \\
\hline
\end{tabular}

De acordo com esta tabela, podemos perceber que o valor de acurácia obtido neste estudo foi menor que o trabalho de Maity et al. 2020. Contudo, vale ressaltar que esses autores não utilizaram todas as imagens dos $P$. falciparum e $P$. vivax para segmentação e classificação do Plasmodium. Os autores usaram as imagens que possuíam apenas uma única fase do parasita por imagem, deixando sem usar as que apresentam múltiplas fases do parasita.

O trabalho de Rahman et al. 2021 aplicou o conjunto de dados completo, com as 210 imagens que possuem os quatro tipos do parasita Plasmodium. Os autores realizaram a binarização das células, não importando o seu tipo, e depois realizaram a classificação em infectado e não infectado.

\section{Conclusão e Trabalhos Futuros}

Neste trabalho, realizamos uma análise comparativa das versões do modelo YOLO (YOLOv4, Scaled-YOLOv4 e YOLOv5) para a detecção do parasita Plasmodium da malária e a sua classificação nas espécies facilparum, vixax, malariae e ovale, em imagens de esfregaço de sangue fino. Os modelos alcançaram excelentes resultado em precisão e tempo de execução nos dois testes que foram realizados. Contudo, a YOLOv5 superou todos os outros modelos aplicados, de acordo com as métricas de avaliação utilizadas.

Dessa forma, conlui-se que os resultados dos testes realizados demostraram a viabilidade e eficácia da utilização da YOLOv5, para detectar e localizar parasitas da malária em imagens de esfregaço de sangue fino com alta precisão e sensibilidade. 
Para abordagens futuras, iremos aplicar esses modelos em um número mais significativo de imagens. Também serão utilizados outros modelos para fins comparativos. Apesar dos bons resultados pretende-se implementar modificações na arquitetura com base nas características específicas das imagens de parasita.

\section{Referências}

Abdurahman, F., Fante, K. A., and Aliy, M. (2021). Malaria parasite detection in thick blood smear microscopic images using modified yolov3 and yolov4 models. BMC bioinformatics, 22(1):1-17.

Al-Masni, M. A., Al-Antari, M. A., Park, J.-M., Gi, G., Kim, T.-Y., Rivera, P., Valarezo, E., Choi, M.-T., Han, S.-M., and Kim, T.-S. (2018). Simultaneous detection and classification of breast masses in digital mammograms via a deep learning yolo-based cad system. Computer methods and programs in biomedicine, 157:85-94.

Alqudah, A., Alqudah, A. M., and Qazan, S. (2020). Lightweight deep learning for malaria parasite detection using cell-image of blood smear images. Revue d'Intelligence Artificielle, 34(5):571-576.

Bochkovskiy, A., Wang, C.-Y., and Liao, H.-Y. M. (2020). Yolov4: Optimal speed and accuracy of object detection. arXiv preprint arXiv:2004.10934.

Chibuta, S. and Acar, A. C. (2020). Real-time malaria parasite screening in thick blood smears for low-resource setting. Journal of digital imaging, pages 1-13.

Cruz, D., Claro, M., Veras, R., Vogado, L., Portela, H., Moura, N., and Luz, D. (2020). P-fidenet: Plasmodium falciparum identification neural network. In International Symposium on Visual Computing, pages 369-380. Springer.

Lin, T.-Y., Maire, M., Belongie, S., Hays, J., Perona, P., Ramanan, D., Dollár, P., and Zitnick, C. L. (2014). Microsoft coco: Common objects in context. In European conference on computer vision, pages 740-755. Springer.

Liu, S., Qi, L., Qin, H., Shi, J., and Jia, J. (2018). Path aggregation network for instance segmentation. In Proceedings of the IEEE conference on computer vision and pattern recognition, pages $8759-8768$.

Ljosa, V., Sokolnicki, K. L., and Carpenter, A. E. (2012). Annotated high-throughput microscopy image sets for validation. Nature methods, 9(7):637-637.

Loddo, A., Di Ruberto, C., Kocher, M., and Prod'Hom, G. (2018). Mp-idb: The malaria parasite image database for image processing and analysis. In Sipaim-Miccai Biomedical Workshop, pages 57-65. Springer.

Maity, M., Jaiswal, A., Gantait, K., Chatterjee, J., and Mukherjee, A. (2020). Quantification of malaria parasitaemia using trainable semantic segmentation and capsnet. Pattern Recognition Letters, 138:88-94.

Maqsood, A., Farid, M. S., Khan, M. H., and Grzegorzek, M. (2021). Deep malaria parasite detection in thin blood smear microscopic images. Applied Sciences, 11(5):2284.

Nie, Y., Sommella, P., O’Nils, M., Liguori, C., and Lundgren, J. (2019). Automatic detection of melanoma with yolo deep convolutional neural networks. In E-Health and Bioengineering Conference, pages 1-4. 
Organization, W. H. et al. (2020). World malaria report 2020: 20 years of global progress and challenges.

Peñas, K. E. D., Rivera, P. T., and Naval, P. C. (2017). Malaria parasite detection and species identification on thin blood smears using a convolutional neural network. In 2017 IEEE/ACM International Conference on Connected Health: Applications, Systems and Engineering Technologies, pages 1-6.

Rahman, A., Zunair, H., Reme, T. R., Rahman, M. S., and Mahdy, M. (2021). A comparative analysis of deep learning architectures on high variation malaria parasite classification dataset. Tissue and Cell, 69:101473.

Rajaraman, S., Antani, S. K., Poostchi, M., Silamut, K., Hossain, M. A., Maude, R. J., Jaeger, S., and Thoma, G. R. (2018). Pre-trained convolutional neural networks as feature extractors toward improved malaria parasite detection in thin blood smear images. PeerJ, 6:e4568.

Ramarolahy, R., Gyasi, E., and Crimi, A. (2020). Classification and generation of microscopy images with plasmodium falciparum via artificial neural networks. bioRxiv.

Redmon, J., Divvala, S., Girshick, R., and Farhadi, A. (2016). You only look once: Unified, real-time object detection. In Proceedings of the IEEE conference on computer vision and pattern recognition, pages 779-788.

Somasekar, J., Reddy, B., Reddy, E., and Lai, C. (2011). Computer vision for malaria parasite classification in erythrocytes. International Journal on Computer Science and Engineering, 3(6):2251-2256.

Ünver, H. M. and Ayan, E. (2019). Skin lesion segmentation in dermoscopic images with combination of yolo and grabcut algorithm. Diagnostics, 9(3):72.

Wang, C.-Y., Bochkovskiy, A., and Liao, H.-Y. M. (2020). Scaled-yolov4: Scaling cross stage partial network. arXiv preprint arXiv:2011.08036.

Wu, D., Lv, S., Jiang, M., and Song, H. (2020). Using channel pruning-based yolo v4 deep learning algorithm for the real-time and accurate detection of apple flowers in natural environments. Computers and Electronics in Agriculture, 178:105742.

Yang, F., Quizon, N., Yu, H., Silamut, K., Maude, R. J., Jaeger, S., and Antani, S. (2020a). Cascading yolo: automated malaria parasite detection for plasmodium vivax in thin blood smears. In Medical Imaging 2020: Computer-Aided Diagnosis, volume 11314, page 113141Q. International Society for Optics and Photonics.

Yang, G., Feng, W., Jin, J., Lei, Q., Li, X., Gui, G., and Wang, W. (2020b). Face mask recognition system with yolov5 based on image recognition. In 2020 IEEE 6th International Conference on Computer and Communications, pages 1398-1404. IEEE.

Zhou, F., Zhao, H., and Nie, Z. (2021). Safety helmet detection based on yolov5. In 2021 IEEE International Conference on Power Electronics, Computer Applications, pages 6-11. 